\title{
Organisierende Zentren am Beispiel einer Kampagne zum Nahverkehr in Kassel
}

sierung verändert die politische Landschaft

Kommentare von: Workers Center München, Kotti\&Co Berlin, Rothe Ecke Kassel

Replik von:

Robert Maruschke

\author{
Rothe Ecke Kassel
}

Im Jahr 2014 trafen sich vier Menschen in Kassel, die alle in verschiedensten gewerkschaftlichen Initiativen und selbstorganisierten politischen Projekten tätig waren. Angeregt von Erfahrungen in den USA, entschlossen wir uns den Stadtteilladen „Rothe Ecke“ im Stadtteil Rothenditmold zu gründen. Ausgangspunkt war, dass wir raus aus den linken Milieus und rein in die Nachbarschaft und unter die Kolleg_innen wollten. Nach unserer Vorstellung soll die Rothe Ecke ein Raum sein, der es Menschen erleichtert, sich gemeinsam mit anderen bei Konfrontationen mit Jobcenter, Vermieter_in, Vorgesetzten oder der Stadt zu organisieren und Infrastruktur und Wissen zur Verfügung stellt, um kollektive Interessen erfolgreicher durchzusetzen. Den Stadtteil wählten wir, da wir teilweise bereits dort lebten und weil in dem ehemaligen Arbeiterstadtteil diejenigen wohnen, für die ein gesellschaftlicher Wandel am drängendsten ist: Prekäre, Arbeitslose, Junge, Alleinerziehende, arbeitende Rentner_innen, Migrant_innen... Sie sind die Ersten, die Armut, prekären Lebensrealitäten, schikanösen Arbeitsbedingungen und Rassismus ausgesetzt sind. Also mieteten wir ein ehemaliges Ladengeschäft mit einem Raum und vielen Schaufenstern, was wir durch Spenden finanzierten. Dann begannen wir damit, verschiedene Ansätze auszuprobieren, wie wir vor Ort transformatives Organizing über Veranstaltungen, die Gründung einer Jobcenter-Initiative, Spieleabende und andere Dinge umsetzen könnten. Wir waren schnell ein Treffpunkt für einzelne Betriebsgruppen (Call Center, Post, Taxifahrer_innen...), die in den Räumen von Gruppen Beratung erhielten, dort bei sozialen Events andere Leute trafen oder einfach einen Raum als Treffpunkt brauchten. Dabei war der Raum wertvoll sowohl für Betriebsgruppen als auch als Begegnungsort verschiedener Beschäftigtengruppen. In dieser Zeit hat er allerdings eher wie ein Workers Center funktioniert.

Dadurch konnten wir existierende Gruppen unterstützen und vernetzen, waren aber nicht dazu in der Lage, unsere eigenen Kräfte, also unser Kernteam, aufbauen und fühlten uns deshalb lange nicht kampagnenfähig. Im Sommer 2015 wurde dann bekannt, dass in Kassel Kürzungen im öffentlichen Nahverkehr anstehen und zum Teil ganze Buslinien gestrichen werden sollen. Diese Umstrukturierung wurde von einem Beteiligungsverfahren 
begleitet, bei dem absehbar war, dass es darauf abzielt, größere Widerstände abzuwiegeln oder einzubinden. So war klar, dass nach diesem Verfahren zwar Änderungen bei einzelnen Linien möglich waren, aber damit nur die Kürzungen an andere Stellen verschoben werden, statt diese grundsätzlich in Frage zu stellen.

Die Herausforderung lag also darin, in unserem Stadtteil das vorherrschende Gefühl der Ohnmacht aufzubrechen und gleichzeitig die Änderungen grundsätzlich anzugreifen, um nicht in die ,Mitmachfalle‘ zu tappen. So wollten wir erreichen, dass auch nach der Überarbeitung der Sparmaßnahmen möglichst viele der Beteiligten dabei bleiben und keine Kürzungen akzeptieren würden.

Wir haben unsere Strategie auf zwei Ebenen entwickelt. Einerseits begannen wir direkt bei uns im Stadtteil mit dem Einsatz für eine konkrete Buslinie, andererseits betrieben wir Bündnisarbeit mit Menschen und Verbänden aus der ganzen Stadt, um die verschiedenen Kritiker_innen an der Netzreform zusammen zu führen und, statt nur einzelne Buslinien zu fokussieren, den Blick auf den gesamten ÖPNV zu lenken. So wollten wir anstelle von Fragen der Wirtschaftlichkeit die Bedürfnisse der Menschen ins Zentrum stellen. Konkrete Wünsche nach Verbesserung versuchten wir immer mit großen, allgemeinen Forderungen zu verbinden, hinter denen wir als Gruppe auch tatsächlich stehen können.

Als Höhepunkt auf halber Strecke, also auf dem ersten Abschnitt der Auseinandersetzung bis zur Überarbeitung des Verkehrsnetzes, organisierten wir eine Woche vor der Kommunalwahl strategisch günstig eine gemeinsame Demonstration, bei der wir alle bis dahin aufgekommenen Forderungen vertraten: günstigere Tickets, Ausbau des Nahverkehrs, bessere Arbeitsbedingungen für die Beschäftigten. Die Demonstration war ein groBer gemeinsamer Erfolg. Denn für viele war das die erste selbstorganisierte Demonstration und viele der Redebeiträge wurden von Frauen gehalten, da besonders Mütter im Bündnis aktiv waren. Dadurch, dass die Organisation nicht allein den Routinierten überlassen wurde, flossen neue Ideen ein und die Demo wurde sehr lebendig. Gerade diejenigen, für die dies ihre erste Demo war, glänzten mit fundierten und begeisternden Redebeiträgen, die plastisch werden ließen, was es für die einzelnen Menschen bedeutet, wenn der Nahverkehr eingeschränkt wird.

Bei uns im Stadtteil begannen wir mit einer Unterschriftenliste, die wir im ganzen Stadtteil auslegten und für die jeder direkt an Haltestellen und im Bus sammeln konnte. Dabei führten wir zahlreiche Einzelgespräche und wir trafen auf eine große Wut und Empörung, da die Kürzungen als weiterer Angriff auf Menschen mit wenig Geld wahrgenommen wurden und die Menschen schnell das konkrete Thema der Mobilität mit allgemeinpolitischen Diskussionen verknüpften.

Jede Woche trafen wir uns für eine Stunde als organisierender Kern in einem kleinen Kreis mit Nachbar_innen. Durch diese kurzen, regelmäßigen Treffen und das gemeinsame Feiern der kleinen und größeren Erfolge sind wir zusammengewachsen.

Wir versuchen unsere Treffen möglichst offen zu gestalten und nicht nur für Absprachen zu nutzen, sondern auch mit direkten Aktivitäten zu verknüpfen: etwa Infoständen draußen, Malen von Transparenten und so weiter. 
Nicht alles gelingt beim ersten Mal. Vor allem wird es bei begrenzten Kapazitäten schwierig, den Balanceakt zu finden zwischen dem Gang in die Breite (also jeden Tag auf die Straße zu gehen, um neue Menschen zu kontaktieren) und dem in die Tiefe (also Zeit haben, die Einzelnen kennen zu lernen und sich über transformatives Organizing und die größere Vision auszutauschen). Den Schritt vom Aktivisten zur Organizerin möchte dabei nicht jede_r neu dazu Gekommene gleich vollziehen. Umso bedeutender war es auch hier, Menschen vor Aktionen direkt anzurufen und zum Beispiel gemeinsam in der Nachbarschaft zu grillen.

Bei den wöchentlichen Treffen sind wir ein kleiner Kreis. Wir haben aber auch gelernt, die Anzahl der Teilnehmer_innen an einem Treffen nicht zum wichtigsten Maßstab zu machen. Nicht immer klappt es zeitlich - manche wollen nicht zu Treffen gehen, sondern bevorzugen Aktionen.

Die in unserem Schaufenster ausgehängten Informationen werden von vielen Menschen gelesen. Für Bildungsarbeit haben wir nur begrenzt Formate gefunden. Wichtig für uns war dabei, dass man sich immer wieder klarmacht, dass Bildung nicht nur im Seminarrahmen stattfindet. Oft besteht Bildung auch aus Diskussionen, wenn man abends zusammensitzt, oder zu zweit Plakate aufhängt, weil dann ein Rahmen geschaffen ist, innerhalb dessen jeder offen Fragen stellen kann. Wichtig war auch, zu sehen, dass wir Bildung nicht immer selbst organisieren müssen. Einmal waren wir etwa gemeinsam bei einer Veranstaltung zum GdL-Streik und konnten auch direkt die Busfahrt dorthin für Gespräche mit anderen Busnutzer_innen nutzen.

Wir spielten des Öfteren durch, wie der Gegner, beziehungsweise in diesem Fall die kommunal Verantwortlichen und die in den Verkehrsbetrieben, reagieren könnten. So bereiteten wir uns auf die nächsten Schritte vor. Zumindest in unserer Gruppe und dem stadtweiten Bündnis ist nun klar, dass wir weiter gegen die Kürzungen und für den Ausbau eines bezahlbaren ÖPNV aktiv bleiben werden, auch nachdem es uns gelungen ist, die Streichung unserer konkreten Buslinie in einer Überarbeitung des Entwurfs zu verhindern. In diesem Prozess arbeiten wir daran neue Menschen einzubinden, Menschen zu finden, die sich dafür entscheiden, Organizer_innen zu werden.

Um also auf das Verhältnis von Organisation und spontaner Bewegung zurück zu kommen: Spontan kam es zu Protesten gegen die Umstrukturierung im Nahverkehr. Dass diese vereinzelten Proteste aber zusammengeführt, stabilisiert und mit einer gesellschaftlichen Frage verknüpft wurden, ist auch das Ergebnis von Verbundenheit, gegenseitigem Lernen und des Wirkens transformativer Kräfte als organisierenden Faktoren. Dass dabei auch gerade für einen nichtbürgerlichen Stadtteil etwas gewonnen wurde und hier die Erfahrung eines erfolgreichen gemeinsamen Widerstands gemacht werden konnte, ist das Ergebnis der Arbeit von anfangs nicht mehr als vier Menschen, die sich bewusst dafür entschieden haben, eine Kampagne zu starten. Ob sich diese Erfahrungen nun auf andere Politikfelder übertragen und in den vorhandenen vertiefen lassen, wird in der Zukunft zu sehen sein.

\section{Autor_innen}


